\title{
Prevalence of vitamin D deficiency in pregnancy among Indian population: A systematic review
}

\section{Akanksha Bhatnagar and Kusum Mittal}

See end of the paper for authors' affiliations Akanksha Bhatnagar

College of Social Science and Humanities, Udaipur (Rajasthan) India

Email : sbakanksha@gmail.com

Received: 14.01.2019; Accepted: 22.05.2019

DABSTRACT : The prevalence of vitamin D deficiency (VDD) is increasing worldwide among all age groups including pregnant females. In our manuscript, we aim to review the existing literature to assess the prevalence of VDD among pregnant females in Indian population and to identify risk factors associated with it. We performed a systematic literature search of PubMed upto October 2018 for relevant articles. We found that prevalence ranged from 56 per cent to 96 per cent. Most of the studies found positive correlation between sun exposure and serum vitamin D level. Four studies found higher prevalence of VDD during winters while one study found higher prevalence during summers. Prevalence was similar among various religions. One study reported lower prevalence among non-vegetarians. Two studies showed no association of daily calcium intake and maternal serum vitamin D level while one study found low intake is associated with low vitamin D level. We conclude that even after adequate sunlight availability in India the prevalence of VDD is very high but further studies are required on vitamin D supplementation to understand its effect on prevention of VDD.

KEY WORDS: Vitamin D deficiency, Pregnant females, Prevalence, Hypovitaminosis, Risk factors

- HOW TO CITE THIS PAPER : Bhatnagar, Akanksha and Mittal, Kusum (2019). Prevalence of vitamin D deficiency in pregnancy among Indian population: A systematic review. Asian J. Home Sci., 14 (1) : 205210, DOI: 10.15740/HAS/AJHS/14.1/205-210. Copyright@ 2019: Hind Agri-Horticultural Society. 